\title{
KÄHLER-EINSTEIN METRICS ON LOG DEL PEZZO SURFACES IN WEIGHTED PROJECTIVE 3-SPACES
}

\author{
JENNIFER M. JOHNSON AND JÁNOS KOLLÁR
}

A log del Pezzo surface is a projective surface with quotient singularities such that its anticanonical class is ample. Such surfaces arise naturally in many different contexts, for instance in connection with affine surfaces Miyanishi81, moduli of surfaces of general type Alexeev94, 3 and 4 dimensional minimal model program Alexeev93. They also provide a natural testing ground for existence results of Kähler-Einstein metrics. The presence of quotient singularities forces us to work with orbifold metrics, but this is usually only a minor inconvenience. Log del Pezzo surfaces with a Kähler-Einstein metric also lead to SasakianEinstein 5-manifolds by [Boyer-Galicki00].

In connection with Demailly-Kollár99], the authors ran a computer program to find examples of log del Pezzo surfaces in weighted projective spaces. The program examined weights up to a few hundred and produced 3 examples of log del Pezzo surfaces where the methods of [Demailly-Kollár99, §6] proved the existence of a Kähler-Einstein metric.

The aim of this paper is twofold. First, we determine the complete list of anticanonically embedded quasi smooth log del Pezzo surfaces in weighted projective 3-spaces. Second, we improve the methods of [Demailly-Kollár99, 6.10] to prove that many of these admit a Kähler-Einstein metric. The same method also proves that some of these examples do not have tigers (in the colorful terminology of Keel-McKernan99).

Higher dimensional versions of these results will be considered in a subsequent paper.

Definition 1. For positive integers $a_{i}$ let $\mathbb{P}\left(a_{0}, a_{1}, a_{2}, a_{3}\right)$ denote the weighted projective 3 -space with weights $a_{0} \leq a_{1} \leq a_{2} \leq a_{3}$. (See Dolgachev82 or [Fletcher89 for the basic definitons and results.) We always assume that any 3 of the $a_{i}$ are relatively prime. We frequently write $\mathbb{P}$ to denote a weighted projective 3 -space if the weights are irrelevant or clear from the context. We use $x_{0}, x_{1}, x_{2}, x_{3}$ to denote the corresponding weighted projective coordinates. We let $(i, j, k, \ell)$ be an 
unspecified permutation of $(0,1,2,3) . P_{i} \in \mathbb{P}\left(a_{0}, a_{1}, a_{2}, a_{3}\right)$ denotes the point $\left(x_{j}=x_{k}=x_{\ell}=0\right)$. The affine chart where $x_{i} \neq 0$ can be written as

$$
\mathbb{C}^{3}\left(y_{j}, y_{k}, y_{\ell}\right) / \mathbb{Z}_{a_{i}}\left(a_{j}, a_{k}, a_{\ell}\right)
$$

This shorthand denotes the quotient of $\mathbb{C}^{3}$ by the action

$$
\left(y_{j}, y_{k}, y_{\ell}\right) \mapsto\left(\epsilon^{a_{j}} y_{j}, \epsilon^{a_{k}} y_{k}, \epsilon^{a_{\ell}} y_{\ell}\right)
$$

where $\epsilon$ is a primitive $a_{i}$ th root of unity. The identification is given by $y_{j}^{a_{i}}=x_{j}^{a_{i}} / x_{i}^{a_{j}}$. (1.1) are called the orbifold charts on $\mathbb{P}\left(a_{0}, a_{1}, a_{2}, a_{3}\right)$.

$\mathbb{P}\left(a_{0}, a_{1}, a_{2}, a_{3}\right)$ has an index $a_{i}$ quotient singularity at $P_{i}$ and an index $\left(a_{i}, a_{j}\right)$ quotient singularity along the line $\left(x_{k}=x_{\ell}=0\right)$.

For every $m \in \mathbb{Z}$ there is a rank 1 sheaf $\mathcal{O}_{\mathbb{P}}(m)$ which is locally free only if $a_{i} \mid m$ for every $i$. A basis of the space of sections of $\mathcal{O}_{\mathbb{P}}(m)$ is given by all monomials in $x_{0}, x_{1}, x_{2}, x_{3}$ with weighted degree $m$. Thus $\mathcal{O}_{\mathbb{P}}(m)$ may have no sections for some $m>0$.

2 (Anticanonically embedded quasi smooth surfaces).

Let $X \in\left|\mathcal{O}_{\mathbb{P}}(m)\right|$ be a surface of degree $m$. The adjunction formula

$$
\left.\left.K_{X} \cong \mathcal{O}_{\mathbb{P}}\left(K_{\mathbb{P}}+X\right)\right|_{X} \cong \mathcal{O}_{\mathbb{P}}\left(m-\left(a_{0}+a_{1}+a_{2}+a_{3}\right)\right)\right|_{X}
$$

holds iff $X$ does not contain any of the singular lines. If this condition holds then $X$ is a (singular) del Pezzo surface iff $m<a_{0}+a_{1}+a_{2}+a_{3}$. It is also well understood that from many points of view the most interesting cases are when $m$ is as large as possible. Thus we consider the case $X_{d} \in\left|\mathcal{O}_{\mathbb{P}}(d)\right|$ for $d=a_{0}+a_{1}+a_{2}+a_{3}-1$. We say that such an $X$ is anticanonically embedded.

Except for the classical cases

$$
\left(a_{0}, a_{1}, a_{2}, a_{3}\right)=(1,1,1,1),(1,1,1,2) \quad \text { or }(1,1,2,3),
$$

$X$ is not smooth and it passes through some of the vertices $P_{i}$. Thus the best one can hope is that $X$ is smooth in the orbifold sense, called quasi smooth. At the vertex $P_{i}$ this means that the preimage of $X$ in the orbifold chart $\mathbb{C}^{3}\left(y_{j}, y_{k}, y_{\ell}\right)$ is smooth. In terms of the equation of $X$ this is equivalent to saying that

For every $i$ there is a $j$ and a monomial $x_{i}^{m_{i}} x_{j}$ of degree $d$. (2.1) Here we allow $j=i$, corresponding to the case when the general $X$ does not pass through $P_{i}$. The condition that $X$ does not contain any of the singular lines is equivalent to

$$
\text { If }\left(a_{i}, a_{j}\right)>1 \text { then there is a monomial } x_{i}^{b_{i}} x_{j}^{b_{j}} \text { of degree } d \text {. (2.2) }
$$


Finally, if every member of $\left|\mathcal{O}_{\mathbb{P}}(d)\right|$ contains a coordinate axis $\left(x_{k}=\right.$ $x_{\ell}=0$ ) then the general member should be smooth along it, except possibly at the vertices. That is

For every $i, j$, either there is a monomial $x_{i}^{b_{i}} x_{j}^{b_{j}}$ of degree $d$, or there are monomials $x_{i}^{c_{i}} x_{j}^{c_{j}} x_{k}$ and $x_{i}^{d_{i}} x_{j}^{d_{j}} x_{\ell}$ of degree $d$.

The computer search done in connection with Demailly-Kollár99 looked at values of $a_{i}$ in a certain range to find the $a_{i}$ satisfying the constraints (2.1-3). This approach starts with the $a_{i}$ and views (2.1-3) as linear equations in the unknowns $m_{i}, b_{i}, c_{i}, d_{i}$. In order to find all solutions, we change the point of view.

3 (Description of the computer program).

We consider (2.1) to be the main constraint, the $m_{i}$ as coefficients and the $a_{i}$ as unknowns. The corresponding equations can then be written as a linear system

$$
(M+J+U)\left(\begin{array}{llll}
a_{0} & a_{1} & a_{2} & a_{3}
\end{array}\right)^{t}=\left(\begin{array}{llll}
-1 & -1 & -1 & -1
\end{array}\right)^{t}
$$

where $M=\operatorname{diag}\left(m_{0}, m_{1}, m_{2}, m_{3}\right)$ is a diagonal matrix, $J$ is a matrix with all entries -1 and $U$ is a matrix where each row has 3 entries $=0$ and one entry $=1$. It is still not easy to decide when such a system has positive integral solutions, but the main advantage is that some of the $m_{i}$ can be bounded a priori.

Consider for instance $m_{3}$. The relevant equation is

$$
m_{3} a_{3}+a_{j}=a_{0}+a_{1}+a_{2}+a_{3}-1 .
$$

Since $a_{3}$ is the biggest, we get right away that $1 \leq m_{3} \leq 2$. Arguing inductively with some case analysis we obtain that

2. either $2 \leq m_{2} \leq 4$ and $2 \leq m_{3} \leq 10$,

3 . or the $a_{i}$ are in a series $(1, a, b, b$,$) with a \mid 2 b-1$. The latter satisfy (2).2) only for $a=b=1$.

Thus we have only finitely many possibilities for the matrix $U$ and the numbers $m_{1}, m_{2}, m_{3}$. Fixing these values, we obtain a linear system

$$
(M+J+U)\left(\begin{array}{llll}
a_{0} & a_{1} & a_{2} & a_{3}
\end{array}\right)^{t}=\left(\begin{array}{llll}
-1 & -1 & -1 & -1
\end{array}\right)^{t},
$$

where the only variable coefficient is the upper left corner of $M$. Solving these formally we obtain that

$$
a_{0}=\frac{\gamma_{0}}{m_{0} \alpha+\beta}
$$

where $\alpha, \beta, \gamma_{0}$ depend only on $U$ and $m_{1}, m_{2}, m_{3}$. $a_{0}$ is supposed to be a positive integer, thus if $\alpha \neq 0$ then there are only finitely many possibilities for $m_{0}$. Once $m_{0}$ is also fixed, the whole system can be 
solved and we check if the $a_{i}$ are all positive integers. We get 1362 cases.

If $\alpha=0$ but $\beta \neq 0$ then the general solution of the system has the form

$$
a_{0}=\frac{\gamma_{0}}{\beta}, a_{i}=\frac{m_{0} \delta_{i}+\gamma_{i}}{\beta} \text { for } \quad i=1,2,3 .
$$

These generate the series of solutions, 405 of them. Finally, with some luck, the case $\alpha=\beta=\gamma_{0}=0$ never occurs, so we do not have to check further.

The resulting solutions need considerable cleaning up. Many solutions $a_{0}, a_{1}, a_{2}, a_{3}$ occur multiply and we also have to check the other conditions (2.2-3). At the end we get the complete list, given in (8).

The computer programs are available at

www . math.princeton.edu/ jmjohnso/LogDelPezzo

These log del Pezzo surfaces are quite interesting in their own right. Namely, it turns out that for many of them, members of the linear systems $\left|-m K_{X}\right|$ can not be very singular at any point. First we recall the notions log canonical etc. (see, for instance, Kollár-Mori98, 2.3] for a detailed introduction).

Definition 4. Let $X$ be a surface and $D$ a $\mathbb{Q}$-divisor on $X$. Let $g$ : $Y \rightarrow X$ be any proper birational morphism, $Y$ smooth. Then there is a unique $\mathbb{Q}$-divisor $D_{Y}=\sum e_{i} E_{i}$ on $Y$ such that

$$
K_{Y}+D_{Y} \equiv g^{*}\left(K_{X}+D\right) \text { and } g_{*} D_{Y}=D .
$$

We say that $(X, D)$ is canonical (resp. klt, resp. log canonical) if $e_{i} \geq 0$ (resp. $e_{i}>-1$, resp. $e_{i} \geq-1$ ) for every $g$ and for every $i$.

Definition 5. Keel-McKernan99 Let $X$ be a normal surface. A tiger on $X$ is an effective $\mathbb{Q}$-divisor $D$ such that $D \equiv-K_{X}$ and $(X, D)$ is not klt. As illustrated in Keel-McKernan99, the tigers carry important information about birational transformations of log del Pezzo surfaces.

Remark 6. By a result of Shokurov (cf. [Keel-McKernan99, 22.2]), if the log del Pezzo surface $X$ has Picard number 1 and it has a tiger then $\left|\mathcal{O}_{X}\left(-m K_{X}\right)\right| \neq \emptyset$ for some $m=1,2,3,4,6$. The log del Pezzo surfaces in (8) mostly have bigger Picard number. It is quite interesting though that the two results work for almost the same cases.

We use the following sufficient condition to obtain the existence of Kähler-Einstein metrics. 
Theorem 7. Nade190, Demailly-Kollár99 Let $X$ be an $n$ dimensional Fano variety (possibly with quotient singularities). Assume that there is an $\epsilon>0$ such that

$$
\left(X, \frac{n+\epsilon}{n+1} D\right) \quad \text { is klt }
$$

for every effective $\mathbb{Q}$-divisor $D \equiv-K_{X}$. Then $X$ has a Kähler-Einstein metric.

The main result of this note is the following.

Theorem 8. There is an anticanonically embedded quasi smooth log del Pezzo surface $X_{d} \subset \mathbb{P}\left(a_{0}, a_{1}, a_{2}, a_{3}\right)$ iff the $a_{i}$ and $d$ are among the following. The table below also gives our results on the nonexistence of tigers (5) and on the existence of Kähler-Einstein metrics. (Lower case $y$ means that the answer has been previously known.)

$\begin{array}{cccccccc} & a_{0} & a_{1} & a_{2} & a_{3} & d & \text { tiger } & \text { KE metric } \\ \text { Series: } & 2 & 2 k+1 & 2 k+1 & 4 k+1 & 8 k+4 & Y & Y \\ \text { Sporadic: } & 1 & 1 & 1 & 1 & 3 & y & y \\ & 1 & 1 & 1 & 2 & 4 & y & y \\ 1 & 1 & 2 & 3 & 6 & y & y \\ 1 & 2 & 3 & 5 & 10 & y & ? \\ 1 & 3 & 5 & 7 & 15 & y & ? \\ 1 & 3 & 5 & 8 & 16 & y & ? \\ 2 & 3 & 5 & 9 & 18 & ? & ? \\ 3 & 3 & 5 & 5 & 15 & N & Y \\ 3 & 5 & 7 & 11 & 25 & ? & Y \\ 3 & 5 & 7 & 14 & 28 & ? & Y \\ 3 & 5 & 11 & 18 & 36 & ? & Y \\ 5 & 14 & 17 & 21 & 56 & N & Y \\ 5 & 19 & 27 & 31 & 81 & N & Y \\ 5 & 19 & 27 & 50 & 100 & N & Y \\ 7 & 11 & 27 & 37 & 81 & N & Y \\ 7 & 11 & 27 & 44 & 88 & N & Y \\ 9 & 15 & 17 & 20 & 60 & N & y \\ 9 & 15 & 23 & 23 & 69 & N & Y \\ 11 & 29 & 39 & 49 & 127 & N & Y \\ 11 & 49 & 69 & 128 & 256 & N & y \\ 13 & 23 & 35 & 57 & 127 & N & Y \\ 13 & 35 & 81 & 128 & 256 & N & y\end{array}$


Remark 9. The above results hold for every quasi smooth surface with the indicated numerical data.

Near the end of the list there are very few monomials of the given degree and in many cases there is only one such surface up to isomorphism. In some other cases, for instance for the series, there are moduli.

It is generally believed that the algebraic geometry of any given log del Pezzo surface can be understood quite well. There is every reason to believe that all of the remaining cases of (8) can be decided, though it may require a few pages of computation for each of them.

10 (How to check if $(X, D)$ is klt or not?).

The definition (4) requires understanding all resolutions of singularities. Instead, we use the following multiplicity conditions to check that a given divisor is klt. These conditions are far from being necessary.

Let $X$ be a surface with quotient singularities. Let the singular points be $P_{i} \in X$ and we write these locally analytically as

$$
p_{i}:\left(\mathbb{C}^{2}, Q_{i}\right) \rightarrow\left(\mathbb{C}^{2} / G_{i}, P_{i}\right) \cong\left(X, P_{i}\right),
$$

where $G_{i} \subset G L(2, \mathbb{C})$ is a finite subgroup. We may assume that the origin is an isolated fixed point of every nonidentity element of $G_{i}$ (cf. Brieskorn68]). Let $D$ be an effective $\mathbb{Q}$-divisor on $X$. Then $(X, D)$ is klt if the following three conditions are satisfied.

1. (Non isolated non-klt points) $D$ does not contain an irreducible component with coefficient $\geq 1$.

2. (Canonical at smooth points) mult $_{P} D \leq 1$ at every smooth point $P \in X$. This follows from [Kollár-Mori98, 4.5].

3. (Klt at singular points) $\operatorname{mult}_{Q_{i}} D_{i} \leq 1$ for every $i$ where $D_{i}:=$ $p_{i}^{*} D$. This follows from [Kollár-Mori98, 5.20] and the previous case.

In our applications we rely on the following estimate.

Proposition 11. Let $Z \subset \mathbb{P}\left(a_{0}, \ldots, a_{n}\right)$ be a d-dimensional subvariety of a weighted projective space. Assume that $Z$ is not contained in the singular locus and that $a_{0} \leq \cdots \leq a_{n}$. Let $Z_{i} \subset \mathbb{A}^{n}$ denote the preimage of $Z$ in the orbifold chart

$$
\mathbb{A}^{n} \rightarrow \mathbb{A}^{n} / \mathbb{Z}_{a_{i}} \cong \mathbb{P}\left(a_{0}, \ldots, a_{n}\right) \backslash\left(x_{i}=0\right) .
$$

Then for every $i$ and every $p \in Z_{i}$,

$$
\operatorname{mult}_{p} Z_{i} \leq\left(a_{n} \cdots a_{n-d}\right)\left(Z \cdot \mathcal{O}(1)^{d}\right)
$$


Moreover, if $Z \neq\left(x_{0}=\cdots=x_{n-d-1}=0\right)$ then we have a stronger inequality

$$
\operatorname{mult}_{p} Z_{i} \leq\left(a_{n} \cdots a_{n-d+1} a_{n-d-1}\right)\left(Z \cdot \mathcal{O}(1)^{d}\right) .
$$

Proof. Let $0 \in C(Z) \subset \mathbb{A}^{n+1}$ denote the cone over $Z$ with vertex 0 . $Z_{i}$ can be identified with the hyperplane section $C(Z) \cap\left(x_{i}=1\right)$. The multiplicty of a point is an upper semi continuous function on a variety, thus it is sufficient to prove that

$$
\operatorname{mult}_{0} C(Z) \leq\left(a_{n} \cdots a_{n-d}\right)\left(Z \cdot \mathcal{O}(1)^{d}\right) .
$$

This is proved by induction on $\operatorname{dim} Z$.

If $C(Z)$ is not contained in the coordinate hyperplane $\left(x_{i}=0\right)$, then write

$$
Z \cap\left(x_{i}=0\right)=\sum_{j} m_{j} Y_{j} \subset \mathbb{P}\left(a_{0}, \ldots, a_{n}\right) .
$$

Next we claim that

$$
\begin{aligned}
\sum_{j} m_{j}\left(Y_{j} \cdot \mathcal{O}(1)^{d-1}\right) & =a_{i}\left(Z \cdot \mathcal{O}(1)^{d}\right) \text { and } \\
\sum_{j} m_{j} \operatorname{mult}_{0} C\left(Y_{j}\right) & \geq \operatorname{mult}_{0} C(Z) .
\end{aligned}
$$

The first of these is the associativity of the intersection product, and the second is a consequence of the usual estimate for the intersection multiplicty (cf. [Fulton84, 12.4]) applied to $C(Z),\left(x_{i}=0\right)$ and $d-1$ other general hyperplanes through the origin. (Note that in the first edition of [Fulton84 there is a misprint in (12.4). $\sum_{i=1}^{r} e_{P}\left(V_{i}\right)$ should be replaced by $\prod_{i=1}^{r} e_{P}\left(V_{i}\right)$.) By the inductive assumption mult ${ }_{0} C\left(Y_{j}\right) \leq$ $\left(Y_{j} \cdot \mathcal{O}(1)^{d-1}\right)$, hence mult ${ }_{0} C(Z) \leq\left(Z \cdot \mathcal{O}(1)^{d}\right)$ as claimed.

In most cases, we can even choose $i<n-d$. This is impossible only if $Z \subset\left(x_{0}=\cdots=x_{n-d-1}=0\right)$, but then equality holds.

Corollary 12. Let $X_{d} \subset \mathbb{P}\left(a_{0}, a_{1}, a_{2}, a_{3}\right)$ be a quasismooth surface of degree $d=a_{0}+a_{1}+a_{2}+a_{3}-1$. Then $X$ does not have a tiger if $d \leq a_{0} a_{1}$. If $\left(x_{0}=x_{1}=0\right) \not \subset X$ then $d \leq a_{0} a_{2}$ is also sufficient.

Proof. Assume that $D \subset X_{d}$ is a tiger. We can view $D$ as a $1-$ cycle in $\mathbb{P}\left(a_{0}, a_{1}, a_{2}, a_{3}\right)$ whose degree is

$$
\left(D \cdot \mathcal{O}_{X}(1)\right)=\left(\mathcal{O}_{\mathbb{P}}(d) \cdot \mathcal{O}_{\mathbb{P}}(1) \cdot \mathcal{O}_{\mathbb{P}}(1)\right)=\frac{d}{a_{0} a_{1} a_{2} a_{3}} .
$$

By (11), this implies that the multiplicity of $D_{i}$ (as in (10.3)) is bounded from above by $\frac{d}{a_{0} a_{1}}$ at any point. Thus $(X, D)$ is klt if $d \leq a_{0} a_{1}$.

If $\left(x_{0}=x_{1}=0\right) \not \subset X$ then we can weaken this to $d \leq a_{0} a_{2}$, again by (11). 
Using (7) and a similar argument we obtain the following.

Corollary 13. Let $X_{d} \subset \mathbb{P}\left(a_{0}, a_{1}, a_{2}, a_{3}\right)$ be a quasismooth surface of degree $d=a_{0}+a_{1}+a_{2}+a_{3}-1$. Then $X$ admits a Kähler-Einstein metric if $d<\frac{3}{2} a_{0} a_{1}$. If $\left(x_{0}=x_{1}=0\right) \not \subset X$ then $d<\frac{3}{2} a_{0} a_{2}$ is also sufficient.

\section{4 (Proof of (8))}

The nonexistence of tigers and the existence of a Kähler-Einstein metric in the sporadic examples follows from (12) and (13). There are 5 cases when we need to use that $X$ does not contain the line $\left(x_{0}=x_{1}=0\right)$. This is equivalent to claiming that the equation of $X$ contains a monomial involving $x_{2}, x_{3}$ only. In all 5 cases this is already forced by the condition (2.1).

Assume next that $X$ is one of the series $(2,2 k+1,2 k+1,4 k+1)$. Its equation is a linear combination of terms

$$
x_{0}^{4 k+2}, x_{3}^{2} x_{0}, x_{3}\left(x_{1}+x_{2}\right) x_{0}^{k+1}, g_{4}\left(x_{1}, x_{2}\right), g_{2}\left(x_{1}, x_{2}\right) x_{0}^{2 k+1} .
$$

Moreover, the conditions (2.1-3) imply that the first 2 appear with nonzero coefficient and $g_{4}$ does not have multiple roots.

$$
\left(x_{0}=0\right) \text { intersects } X \text { in a curve } C \text { whith equation }
$$

$$
\begin{aligned}
& \left(q_{8 k+4}\left(x_{1}, x_{2}\right)=0\right) \subset \mathbb{P}^{2}(2 k+1,2 k+1,4 k+1), \quad \text { isomorphic to } \\
& \left(q_{4}\left(x_{1}, x_{2}\right)=0\right) \subset \mathbb{P}^{2}(1,1,4 k+1) .
\end{aligned}
$$

Thus $C$ has 4 irreducible components $C_{1}+C_{2}+C_{3}+C_{4}$ meeting at $P_{3}=(0: 0: 0: 1)$. This shows that $\frac{1}{2} C$ is not klt at $P_{3}$ and $\frac{1}{2} C$ is a tiger on $X$.

Next we prove that $(X, D)$ is log canonical for every effective $\mathbb{Q}$ divisor $D \equiv-K_{X}$. This is stronger than needed in order to apply (7).

Consider the linear system $\mathcal{O}_{\mathbb{P}}(2(2 k+1))$. This is the pull back of $\mathcal{O}(2(2 k+1))$ from the weighted projective plane $\mathbb{P}(2,2 k+1,2 k+$ $1)$. The latter is isomorphic to $\mathbb{P}(2,1,1)$ which is the quadric cone in ordinary $\mathbb{P}^{3}$ and the linear system is the hyperplane sections, thus very ample. Hence for every smooth point $P \in X$ there is a divisor $F \in\left|\mathcal{O}_{X}(2(2 k+1))\right|$ passing through $P$ and not containing any of the irreducible components of $D$. So

$$
\operatorname{mult}_{P} D \leq(D \cdot F)=\frac{2(2 k+1)(8 k+4)}{2(2 k+1)^{2}(4 k+1)}=\frac{4}{4 k+1}<1 .
$$

We are left to deal with the singular points of $X$. These are at $P_{3}=$ $(0: 0: 0: 1)$ and at $P_{a}=(0: a: 1: 0)$ where $a$ is a root of $g_{4}\left(x_{1}, 1\right)$. 
$P_{3}$ is the most interesting. Let $p_{3}:\left(S \cong \mathbb{C}^{2}, Q_{3}\right) \rightarrow\left(X, P_{3}\right)$ be a local orbifold chart. Intersecting $p_{3}^{*} D$ with a general member of the linear system $\left|x_{0}^{2 k+1}, x_{1}^{2}\right|$ we obtain that

$$
\operatorname{mult}_{Q_{3}} p_{3}^{*} D \leq \frac{4 k+1}{2}(D \cdot \mathcal{O}(2(2 k+1))=2 .
$$

This is too big to apply (10.3). Let $\pi: S^{\prime} \rightarrow S$ be the blow up of the origin with exceptional divisor $E$. Then

$$
K_{S^{\prime}}+\alpha E+\pi_{*}^{-1}\left(p_{3}^{*} D\right) \equiv \pi^{*}\left(K_{S}+p_{3}^{*} D\right)
$$

and $\alpha \leq 1$. Using Shokurov's inversion of adjunction (see, for instance Kollár-Mori98, 5.50]) $(X, D)$ is $\log$ canonical at $P_{3}$ if $\left.\pi_{*}^{-1}\left(p_{3}^{*} D\right)\right|_{E}$ is a sum of points, all with coefficient $\leq 1$. In order to estimate these coefficients, we write $D=D^{\prime}+\sum a_{i} C_{i}$ where $D^{\prime}$ does not contain any of the $C_{i}$.

We first compute that

$$
\left(C_{i} \cdot C_{j}\right)=\frac{1}{4 k+1} \quad \text { if } i \neq j, \text { and } \quad\left(C_{i} \cdot \mathcal{O}(1)\right)=\frac{1}{(2 k+1)(4 k+1)} .
$$

From this we obtain that

$$
\left(C_{i} \cdot C_{i}\right)=\left(C_{i} \cdot \mathcal{O}(1)\right)-\sum_{j \neq i}\left(C_{i} \cdot C_{j}\right)=\frac{-(6 k+1)}{(2 k+1)(4 k+1)} .
$$

Thus

$$
\frac{1}{(2 k+1)(4 k+1)}=\left(C_{i} \cdot D\right)=a_{i}\left(C_{i} \cdot C_{i}\right)+\left(\sum_{j \neq i} a_{j}\right)\left(C_{i} \cdot C_{i+1}\right)+\left(C_{i} \cdot D^{\prime}\right)
$$

Multiplying by $(2 k+1)(4 k+1)$ and using that $\sum a_{i} \leq \operatorname{mult}_{Q_{3}} p_{3}^{*} D \leq 2$ and $\left(C_{i} \cdot D^{\prime}\right) \leq(D \cdot D)$ this becomes

$$
1 \leq-(6 k+1) a_{i}+\left(2-a_{i}\right)(2 k+1)+4 \text { which gives } a_{i} \leq \frac{1}{2}+\frac{2}{4 k+1} .
$$

Furthermore,

$$
\operatorname{mult}_{Q_{3}} p_{3}^{*} D^{\prime} \leq \frac{1}{4}\left(p_{3}^{*} D^{\prime} \cdot \sum_{i} p_{3}^{*} C_{i}\right) \leq \frac{4 k+1}{4}(D \cdot \mathcal{O}(2))=\frac{1}{2 k+1} .
$$

Thus we see that

$$
\left.\pi_{*}^{-1}\left(p_{3}^{*} D\right)\right|_{E}=\left.\sum a_{i} \pi_{*}^{-1}\left(p_{3}^{*} C_{i}\right)\right|_{E}+\left.\pi_{*}^{-1}\left(p_{3}^{*} D^{\prime}\right)\right|_{E}
$$

is a sum of 4 distinct points with coefficient $\leq \frac{1}{2}+\frac{2}{4 k+1}$ and another sum of points where the sum of the coefficients is $\leq \frac{1}{2 k+1}$. Since $\frac{1}{2}+$ $\frac{2}{4 k+1}+\frac{1}{2 k+1}<1$, we see that $(X, D)$ is $\log$ canonical at $P_{3}$.

The points $P_{a}$ are easier. Only one of the $C_{i}$ passes through each of them, and the multiplicity of the pull back of $D^{\prime}$ is bounded by $\frac{2 k+1}{4}(D \cdot \mathcal{O}(2))=\frac{1}{4 k+1}$. This shows right away that $(X, D)$ is klt at these points. 
Acknowledgments . We thank Ch. Boyer, J.-P. Demailly and S. Keel for helpful comments and references. Partial financial support was provided by the NSF under grant number DMS-9970855.

\section{REFERENCES}

Alexeev93. V. Alexeev: Two two-dimensional terminations. Duke Math. J. 69 (1993) 527-545.

Alexeev94. V. Alexeev: Boundedness and $K^{2}$ for $\log$ surfaces. Internat. J. Math. 5 (1994) 779-810.

Boyer-Galicki00. C. Boyer and K. Galicki: New Einstein Manifolds in Dimension Five; Manuscript,

Brieskorn68. E. Brieskorn, Rationale Singularitäten komplexer Flächen, Invent. Math. 4 (1968) 336-358

Demailly-Kollár99. J.-P. Demailly and J. Kollár: Semi-continuity of complex singularity exponents and Kähler-Einstein metrics on Fano orbifolds, (to appear)

Dolgachev82. I. Dolgachev, Weighted projective varieties, in: Group actions and vector fields, Springer Lecture Notes in Math. vol. 956, (1982) 34-71

Fletcher89. A.R. Fletcher: Working with weighted complete intersections ; Preprint MPI/89-35, Max-Planck Institut für Mathematik, Bonn, 1989

Fulton84. W. Fulton, Intersection theory, Springer-Verlag, Berlin - New York, 1984, Second ed. 1998

Keel-McKernan99. S. Keel and J. McKernan: Rational curves on quasi-projective surfaces. Mem. Amer. Math. Soc. 140 (1999), no. 669

Kollár97. J. Kollár: Singularities of pairs, Algebraic Geometry, Santa Cruz, 1995; Proceedings of Symposia in Pure Math. vol. 62, AMS, 1997, 221-287

Kollár-Mori98. J. Kollár and S. Mori: Birational geometry of algebraic varieties, Cambridge Univ. Press 1998

Miyanishi81. M. Miyanishi: Noncomplete algebraic surfaces. Lecture Notes in Mathematics, 857. Springer-Verlag, Berlin-New York, 1981.

Nadel90. A.M. Nadel: Multiplier ideal sheaves and Kähler-Einstein metrics of positive scalar curvature; Annals of Math. 132 (1990), 549-596

Princeton University, Princeton NJ 08544-1000

jmjohnso@math.princeton.edu

kollar@math.princeton.edu 